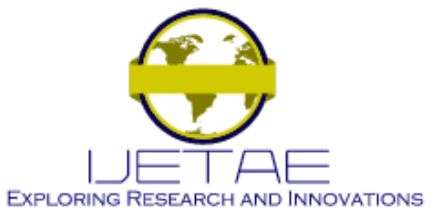

International Journal of Emerging Technology and Advanced Engineering Website: www.ijetae.com (E-ISSN 2250-2459, SCOPUS Indexed Journal, Volume 10, Issue 12, December 2020)

\title{
Analysis of Techniques for On-Line Secondary Path Modeling in ANC Systems
}

\author{
E. O. Lara-Alemán ${ }^{1}$, F. Sandoval-Ibarra ${ }^{2}$, S. Ortega-Cisneros ${ }^{3}$ \\ ${ }^{I}$ Universidad Autónoma de Guadalajara, Av. Patria 1201, 45129 Zapopan, Mexico. \\ ${ }^{2,3}$ Cinvestav-Unidad Guadalajara, Av. Del Bosque 1145, 45017 Zapopan, Mexico.
}

\begin{abstract}
The on-line secondary path modeling of an ANC systems is the most relevant issue. Currently there are algorithms to obtain fast convergence, adequate stability and minimum error. In this article, the properties of these algorithms are analyzed, as well as the architectures for their possible implementation in hardware. The performance comparison is carried out with behavioral simulation, and its suitability for active noise cancellation is evaluated. Based on the results of the analysis, a new algorithm is proposed, suitable for its implementation in hardware. The results obtained present an attenuation of the order of $\mathbf{- 3 1 d B}$, which is the highest attenuation reported so far.
\end{abstract}

Keywords-Active noise, ANC systems, behavioral simulation, digital signal processing, fast convergence.

\section{INTRODUCTION}

Noise damages hearing and as it is happening all over the world, society suffers from acoustic trauma. In practice, because hearing impairment is a health problem, engineering strategies have been developed to minimize the damaging effect of noise on the population. Keep in mind that a noise source emits noise waves that travel to a receiver, and when this is the human being, acoustic trauma occurs if the exposure is for long periods of time. Consequently, who favors the transit of noise is the Communication Channel (CC). Moreover, that fact has favored that the hearing care in the population is to alter, in a controlled way, the CC. This fact requires developing an anti-noise signal, which propagates in the $\mathrm{CC}$, in a way that counteracts the transit of noise waves. From the point of view of Engineering, the signal resulting from this interaction is expected to be of low amplitude. Two types of acoustic noise are considered as case studies, broadband noise and narrowband noise. Broadband noise is random, and its energy is distributed over the entire frequency spectrum. Narrowband noise, on the other hand, is quasiperiodic noise and concentrates a high percentage of its energy at certain frequencies. At present, acoustic noise is controlled with passive and active methods.
Due to the physical dimension that a passive method implies, the active method is more convenient because it includes electroacoustic devices that cancel noise through an anti-noise signal, of equal magnitude to the noise wave but of opposite phase. In this cancellation process, the effectiveness depends on the correct magnitude and the phase of the anti-noise signal generated. This approach, strictly speaking, is called Primary Noise Cancellation, and a DSP is used to generate the anti-noise signal. Furthermore, because the acoustic characteristics of the noise source and the communication channel are timevarying, the noise waves and the anti-noise signal are nonstationary. This characteristic requires that any active noise control system be adaptive.

At present, noise cancellation systems are developed under two approaches, those that use a feedforward control or alternatively those that use a feedback control. In general, the principle of operation is as follows. Using the feedforward control (see Fig. 1), the noise signal is sensed, and the anti-noise signal $y(n)$ is generated. Note that when executing these tasks, the noise $d(n)$ travels a distance $D_{l}$ and there the signals coincide and cancel each other. For this, a microphone (the input transducer) is in position $D=D_{0}$ and the horn (another transducer) is located in $D=D_{l}$. At the point of coincidence, or cancellation zone, it is necessary to feed the controller with the error signal $e(n)$ to modify the value of the coefficients of an adaptive filter and execute its task until the magnitude of the error is minimized. In feedback control, on the other hand, the active controller cancels the noise signal without using a reference signal but by making prediction. In practice, because the cancellation is not always as expected, this method is not analyzed in this contribution.

In recent years, interest has increased in developing active noise controllers, and the trend is to use two channels (see Fig. 2), where $P(z)$ is a digital filter that represents the physical medium through which the noise travels to the noise free zone. 


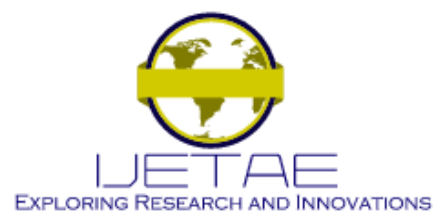

International Journal of Emerging Technology and Advanced Engineering Website: www.ijetae.com (E-ISSN 2250-2459, SCOPUS Indexed Journal, Volume 10, Issue 12, December 2020)

$S(z)$ is another digital filter that models the physical medium through which the anti-noise signal $y^{\prime}(t)$ travels, and travels the distance between the loudspeaker and a second microphone that detects the error $e(t)$. The ultimate goal is that any variation in both CCs is detected promptly and that the coefficients of the digital filters adapt quickly to minimize the error. In addition, in recent years, adaptive algorithms with high convergence speed and greater noise attenuation have been proposed. However, which is the algorithm that effectively offers high speed of convergence, which of these algorithms can be synthesized in a DSP, and which are the evaluation metrics of an active noise controller. In this contribution, these and other questions will be tried to answer based on behavioral results [1].

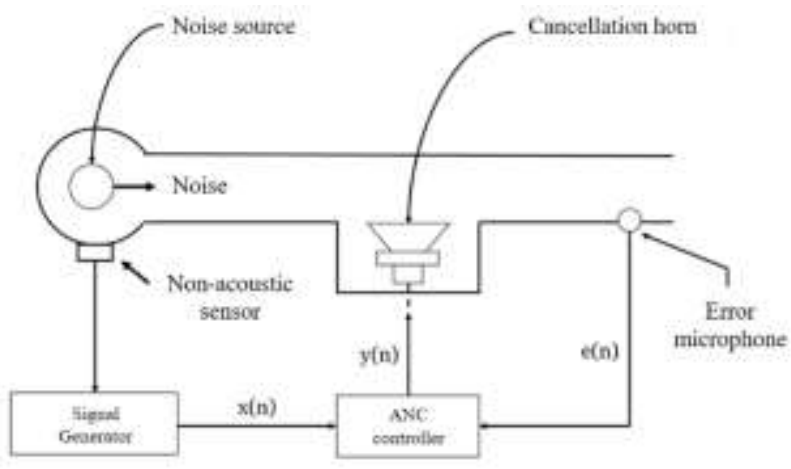

Fig. 1 Narrowband ANC with pre-feed.

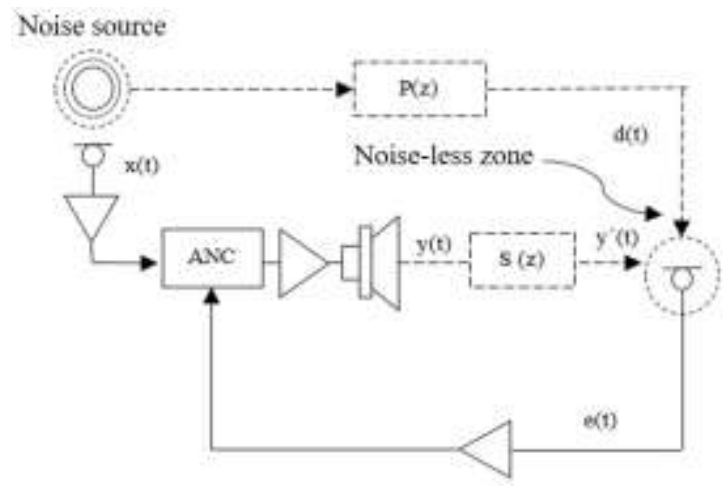

Fig. 2 Communication channels in an ANC system.

This work is organized as follows. In section II the algorithms available in the literature are described, and they are distinguished from each other using various performance characteristics. The evaluation of the secondary CC is also presented in the same section, where the analyzed algorithms are firstly evaluated in terms of the convergence time via behavioral simulation.
The analysis/discussion of results is widely presented in section III, where the authors' proposal resulting from the analysis of the algorithms is included. The conclusions, and future work, are given in Section IV.

\section{STATE Of THE ART}

In an ANC system, the estimation of the CCs is carried out with digital filters, which continuously adjust the coefficients so that the system error decreases and the convergence condition is satisfied. Therefore, when the system converges, the filter coefficients converge to their optimal values. In practice, these coefficients represent both the additive inverse of the primary $\mathrm{CC}$ and the estimate of the secondary CC. Furthermore, it should be noted that the Controller is the adaptive filter that estimates the additive inverse of the primary $\mathrm{CC}$, and its task is performed with adaptive algorithms. Currently, there is a significant number of algorithms to estimate the additive inverse of the first CC. Among the most used algorithms, LMS [2] stands out for its simplicity, there are also its variants that are the LMS family [3], and others of greater complexity, among which the RLS algorithm [4]-[7] stands out.

The location of the controller, in an ANC system, is shown in Fig. 3, and if the estimation of the secondary CC is included as part of the system, the architecture shown in Fig. 4 results, where the performance modeling is with algorithms for adaptive $X$ filters [8]-[10]. The FXLMS algorithm [11], for example, is an adaptive $\mathrm{X}$ algorithm. It is based on a data processing routine that updates the coefficients of the adaptive $\mathrm{W}$ filter using the relation

$$
w(n+1)=w(n)-\mu e(n) x^{\prime}(n)
$$

where $w(n+1)$ is the following coefficient, $w(n)$ is the current coefficient, $\mu$ is the convergence factor, $x^{\prime}(n)$ is the response of the filter $S^{\prime}(z)$ and $e(n)$ is the error; $S^{\prime}(z)$ models the secondary channel. Then, to implement the FXLMS algorithm, the response $S(z)$ of the system is required, that is, the relationship between the signals $y(n)$ and $y^{\prime}(n)$ must be known. Then, once the coefficients of the filter $S^{\prime}(z)$ are known, which is expected to be equivalent to the system $S(z)$, the configuration shown in Fig. 4 would be used, and it is called off-line estimation. In off-line estimation, the transfer function $S(z)$ is estimated first and then active noise cancellation is performed. For practical purposes the following should be discussed. As the horn experiences aging, due to temperature changes and other environmental effects, the offline estimation does not adapt to changes in $S(z)$. 


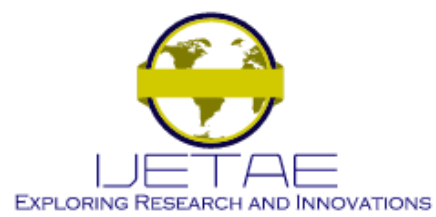

International Journal of Emerging Technology and Advanced Engineering

Website: www.ijetae.com (E-ISSN 2250-2459, SCOPUS Indexed Journal, Volume 10, Issue 12, December 2020)

Therefore, the problem is solved by estimating $S(z)$ at the same time that active noise cancellation occurs. This solution is called online estimation [12]. The idea is to add a random noise generator and a second adaptive filter to the ANC system. However, the trend in the online estimation of the secondary $\mathrm{CC}$ is as shown in Fig. 5, where the random noise allows identifying $S(z)$, and considering that it is added to the signal emitted by the loudspeaker, the filter principal does not execute cancellation. Modifications to the Eriksson method have been proposed from this idea, and in general the objective is to improve the attenuation of the system, achieve higher convergence speed and achieve stability.

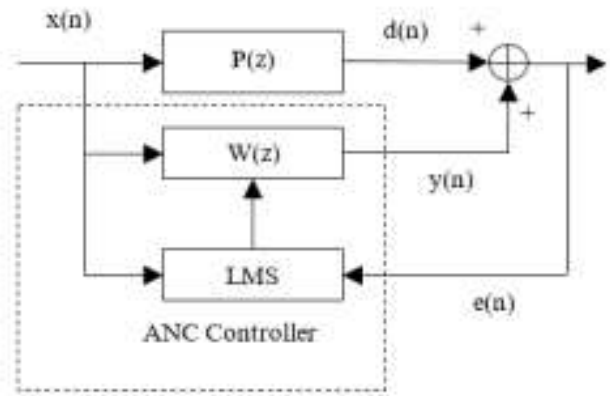

Fig. 3 Controller to estimate the additive inverse of the first $\mathrm{CC}$.

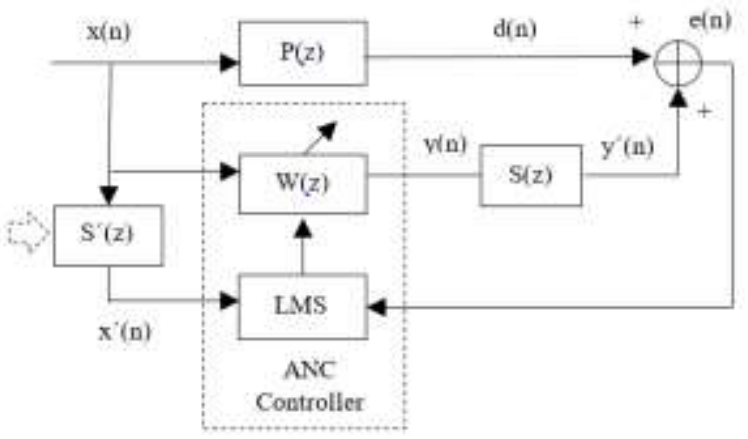

Fig. 4 Estimator for the second CC, Algorithm FxLMS.

\section{A. On-line modeling of the second CC}

With the purpose of canceling noise, and considering that the characteristics can be modified during the operation of the system, the most relevant proposals available in the literature are listed in Table I. Then, to make the performance comparison, each proposal is simulated using behavioral modeling, and proposing that $\mathrm{P}$ (z) and S (z) are FIR filters of length 49 and 17, respectively [13]. Table I also shows the complexity of each proposal, that is, the number of multiplications performed per iteration is shown.
The responses of both the primary $\mathrm{CC}$ and the secondary $\mathrm{CC}$ are shown in Fig. 6 and Fig. 7, respectively, where the solid lines represent both $\mathrm{CCs}$ and the degraded lines are the changes experienced by the channels when $n=5.0 \times 10^{4}$; system sampling was selected at $2 \mathrm{kHz}$. It must be said that a design of experiments was made to obtain the parameters that lead to a high speed of convergence, to a minimum error and to ensure stability. Then, the size of the filters, which estimate the behavior of the secondary CC, $S^{\prime}(z)$, and the controller, $W(z)$, were 17 and 32 coefficients, respectively. For the third filter, $C(z)$, in the case of Zhang's method a 17-coefficient FIR filter was used. In the simulations carried out in this work, the proposals (see Table I) were compared with the parameters shown in Table II. A white noise source, of variance 0.05 , was also used as a training signal to model the secondary CC. Furthermore, two specific cases were defined in the evaluation. In Case 1, the narrowband primary noise cancellation is compared, while in Case 2 the effectiveness of each proposal is considered when random disturbances appear in the CCs and broadband primary noise as well.

Table I

ON-LINE MODELLING OF THE SECOND CC

\begin{tabular}{|c|c|c|c|}
\hline \multirow{2}{*}{ Proposal } & \multicolumn{2}{|c|}{ Algorithm } & \multirow{2}{*}{ \# of operations } \\
\cline { 2 - 3 } & $\mathbf{1}^{\circ}$ CC & $\mathbf{2}^{\circ}$ CC & \\
\hline Eriksson [12] & LMS & LMS & $2 \mathrm{M}+2 \mathrm{~L}$ \\
\hline Zhang [14] & LMS & LMS & $2 \mathrm{M}+2 \mathrm{~L}+2 \mathrm{~N}$ \\
\hline Akhtar [15] & LMS & VSLMS & $2 \mathrm{M}+2 \mathrm{~L}+8$ \\
\hline Davari [16] & LMS & VSLMS & $2 \mathrm{M}+2 \mathrm{~L}+16$ \\
\hline Auxiliary noise [17] & LMS & LMS & $2 \mathrm{M}+2 \mathrm{~L}+1$ \\
\hline Noise programming [18] & VSLM & LMS & $3 \mathrm{M}+2 \mathrm{~L}+4$ \\
\hline Dong [19] & VNLMS & NLMS & $3 \mathrm{M}+3 \mathrm{~L}+2$ \\
\hline
\end{tabular}

Table II

PARAMETERS FOR BEHAVIORAL SIMULATION

\begin{tabular}{|c|l|}
\hline \multirow{2}{*}{ Proposal } & \multicolumn{1}{|c|}{ Parameters } \\
& \\
\hline Eriksson & $\mu_{\mathrm{w}}=1 \times 10^{-4}, \mu_{\mathrm{s}}=1 \times 10^{-2}$ \\
\hline Zhang & $\mu_{\mathrm{w}}=1 \times 10^{-4}, \mu_{\mathrm{s}}=1 \times 10^{-2}, \mu_{\mathrm{c}}=1 \times 10^{-2}$ \\
\hline Akhtar & $\begin{array}{l}\mu_{\mathrm{w}}=1 \times 10^{-4}, \mu_{\mathrm{smin}}=35 \times 10^{-4}, \mu_{\mathrm{smax}}=2 \times 10^{-2}, \\
\lambda=0.9999\end{array}$ \\
\hline Davari & $\begin{array}{l}\mu_{\mathrm{w}}=3 \times 10^{-3}, \mu_{\mathrm{smin}}=2 \times 10^{-2} \mu_{\mathrm{smax}}=7 \times 10^{-2}, \\
\lambda=0.99, \gamma=0.9, \alpha=3 \times 10^{-5}\end{array}$ \\
\hline Auxiliary noise & $\mu_{\mathrm{w}}=1 \times 10^{-5}, \mu_{\mathrm{s}}=1 \times 10^{-2}$ \\
\hline Noise & $\begin{array}{l}\mu_{\mathrm{w}}=1 \times 10^{-6}, \mu_{\mathrm{s}}=1 \times 10^{-2}, \lambda(1)=0.4, \\
\varepsilon=12 \times 10^{-4}, \gamma=0.9999\end{array}$ \\
\hline programming & $\varepsilon=3 \times 10^{-3}, \beta=1$ \\
\hline Dong & $\mu_{\mathrm{w}}=3 \times 10^{-3}, \mu_{\mathrm{s}}=3 \times 1$ \\
\hline
\end{tabular}




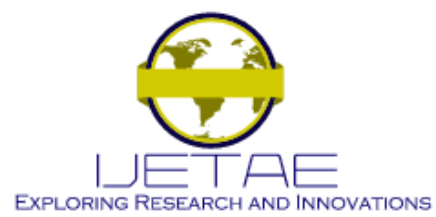

International Journal of Emerging Technology and Advanced Engineering

Website: www.ijetae.com (E-ISSN 2250-2459, SCOPUS Indexed Journal, Volume 10, Issue 12, December 2020)

To show the convergence speed, the accuracy of the secondary CC modeling and the total attenuation of the system are considered. For this, two metrics are considered, the relative modeling error and the root mean square error. The first one is given by

$$
\Delta \mathrm{S}(\mathrm{dB})=10 \log _{10}\left\{\frac{\sum_{\mathrm{i}=0}^{\mathrm{M}-1}\left[\mathrm{~s}_{\mathrm{i}}(\mathrm{n})-\mathrm{s}_{\mathrm{i}}(\mathrm{n})\right]^{2}}{\sum_{\mathrm{i}=0}^{\mathrm{M}-1}\left[\mathrm{~s}_{\mathrm{i}}(\mathrm{n})\right]^{2}}\right\}
$$

where $s_{i}(n)$ and $s^{\prime}{ }_{i}(n)$ are the i-coefficients of the filters $S(z)$ and $S^{\prime}(z)$, respectively; $M$ is the size of the filters. Then, to initialize the values of the coefficients of $S^{\prime}(z)$, $s^{\prime}(0)$, the off-line modeling of the secondary $\mathrm{CC}$ is done. In practice, modeling ends when the error equals $-5 \mathrm{~dB}$.

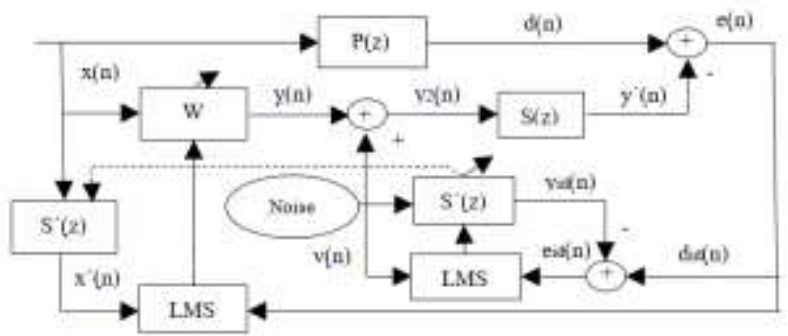

Fig. 5 FxLMS algorithm with on-line estimation, Eriksson's method.

\section{Case 1}

In a practical ANC system, the primary noise signal $x(n)$ consists of periodic narrow band components (such as those produced by motors, compressors, fans, etc.), and for that reason in performance analysis it is considered as primary noise primary up to four components $(100 \mathrm{~Hz}, 200$ $\mathrm{Hz}, 300 \mathrm{~Hz}$, and $400 \mathrm{~Hz}$ ), with a variance set to 2 [1]. In addition, a source of white noise is added to the primary noise signal. The results obtained are shown in Figs. 8 and 9. Note that, in Fig. 8, the Zhang and Dong proposals reach the lowest error in the secondary CC modeling $(-55 \mathrm{~dB}$ and $-49 \mathrm{~dB}$, respectively). However, Dong's proposal requires a longer stabilization time (equivalent to $5 \times 10^{4}$ iterations), which is higher than the $2 \times 10^{3}$ iterations of Zhang's proposal.

Remember that the stabilization time is considered as the time required for the algorithm to reach the lowest error value, the lowest MSE. In other words, if an algorithm is required that adequately estimates the behavior of the secondary CC, Zhang provides the answer. However, the Zhang's proposal is also the one that uses the most computational resources because it includes a third filter. Therefore, it should be noted that the most important metric -in noise cancellation systems- is the final magnitude of the error signal. That metric establishes how much the primary noise signal is attenuated by the system.
The results of the MSE metric, in each proposal, are shown in Fig. 9, where the best performing ones are those that include algorithms that eliminate the source of training noise, such as Davari's proposal, scaled noise and the noise programming one. All three proposals achieve a root mean square error of $-30 \mathrm{~dB}$, compared to $-10 \mathrm{~dB}$ for the rest. Note that Davari's proposal has the highest convergence speed, being $1.2 \times 10^{4}$ iterations compared to $1.7 \times 10^{4}$ and $6.0 \times 10^{4}$ iterations of the scaled noise and noise programming proposals.

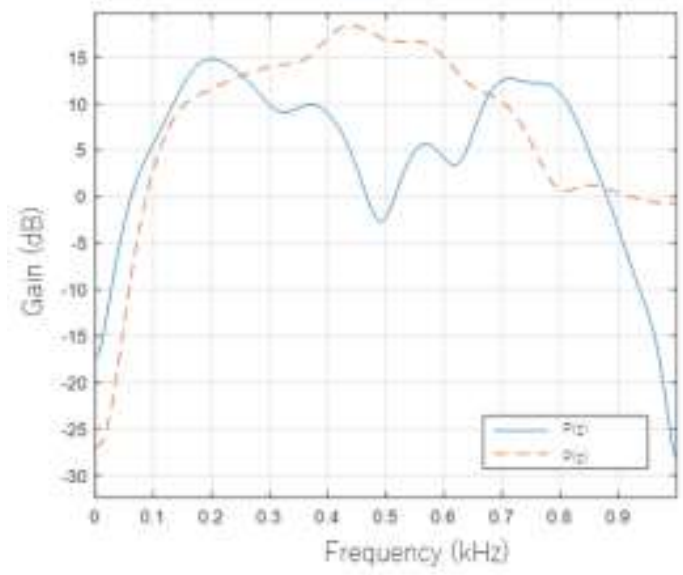

Fig. 6 Response of the primary communication channel, $P(z)$.

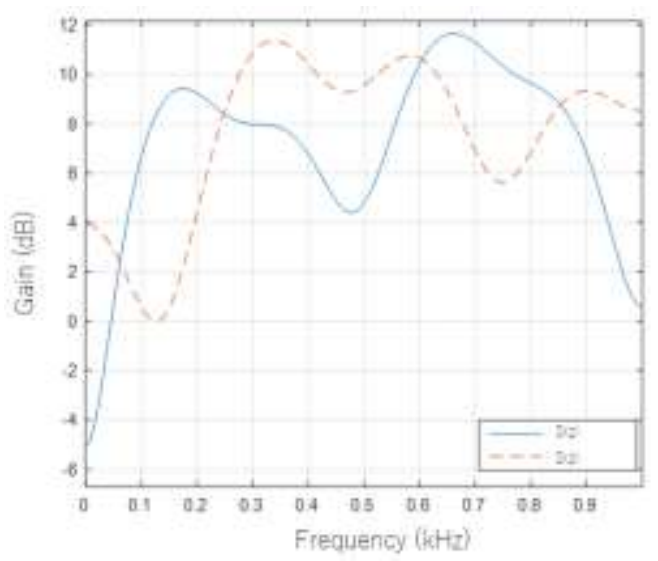

Fig. 7 Response of the secondary communication channel, $S(z)$.

Case 2

Now the primary noise signal is a Gaussian signal (broadband white noise). The variance is set to 1 and is not correlated with the auxiliary white noise. The analysis assumes that the primary and secondary $\mathrm{CC}$ change suddenly during the operation in the iteration $n=5 \times 10^{4}$. 


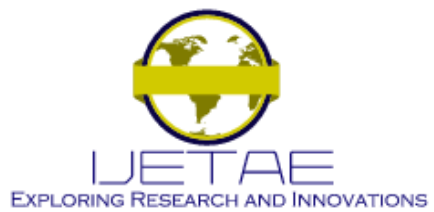

International Journal of Emerging Technology and Advanced Engineering

Website: www.ijetae.com (E-ISSN 2250-2459, SCOPUS Indexed Journal, Volume 10, Issue 12, December 2020)

The results are shown in Fig. 10 and Fig. 11, where the highest speed of convergence corresponds to the Eriksson, Zhang and Akhtar proposals, and at the same time, they are the ones that present the worst performance in the mean square error. When considering primary (broadband) noise, the speed of convergence increases. Regarding modeling error, Zhang and Dong show the best performance, $-43 \mathrm{~dB}$ each one. On the other hand, the one with the lowest MSE value is Davari with a value of $-29 \mathrm{~dB}$ after the disturbance occurred, while the modeling error $(\Delta \mathrm{S})$ is $-41 \mathrm{~dB}$. It can be concluded that the Scaled Noise and Noise Programming proposals are not effective in attenuating broadband noise. The error in the secondary CC modeling is $-35 \mathrm{~dB}$ and $29 \mathrm{~dB}$, respectively, while the other proposals present modeling errors less than $-40 \mathrm{~dB}$. Therefore, the proposals analyzed effectively ensure stability, but Davari's proposal is the one with the best performance in MSE, while its speed of convergence and modeling error can be considered acceptable. Table III shows the results obtained from all proposals.

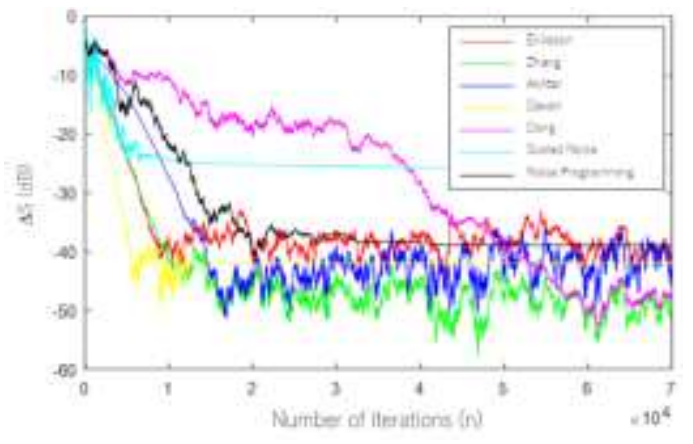

Fig. 8 Simulation results for Case 1, modeling error.

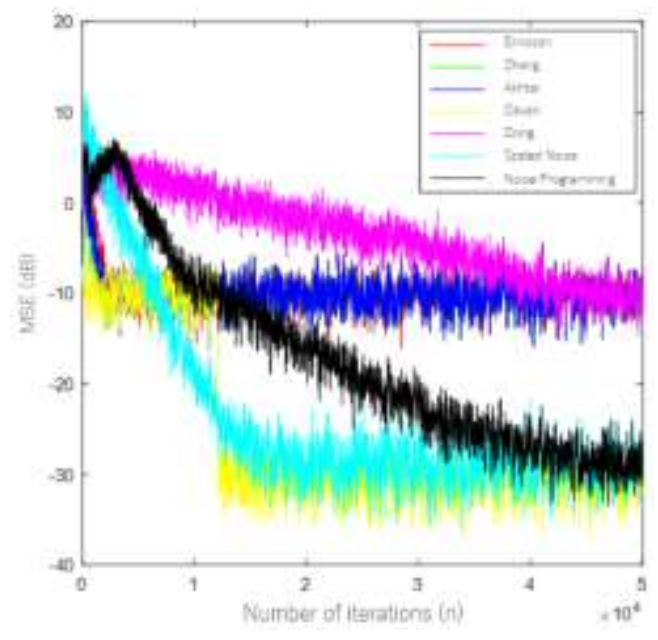

Fig. 9 Simulation results for Case 1, MSE.

\section{DISCUSSION OF RESULTS}

While one of the goals in active noise cancellation is the modeling of the secondary $\mathrm{CC}$, the most important goal is noise attenuation is to decrease the value of the error signal $e(n)$. The online modeling of the secondary $\mathrm{CC}$ is relevant for the good performance of the system, and more because it favors adaptation to the variable conditions of the secondary CC. While one of the goals in active noise cancellation is the modeling of the secondary $\mathrm{CC}$, the most important goal is noise attenuation, that is, to decrease the value of the error signal $e(n)$. The online modeling of the secondary $\mathrm{CC}$ is relevant for the good performance of the system, and more because it favors adaptation to the variable conditions of the secondary $\mathrm{CC}$. This fact explains why methods have been proposed to model secondary CC online, and why it is justified to include a training signal (usually a Gaussian white noise source).

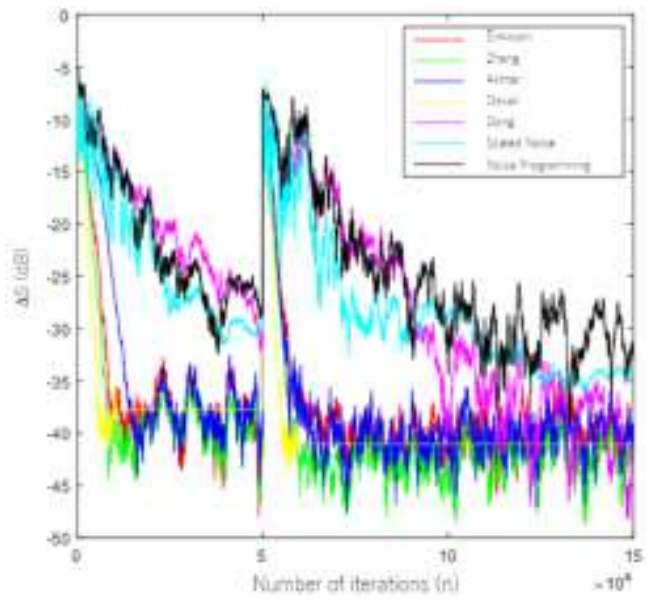

Fig. 10 Simulation results for Case 2, modeling error.

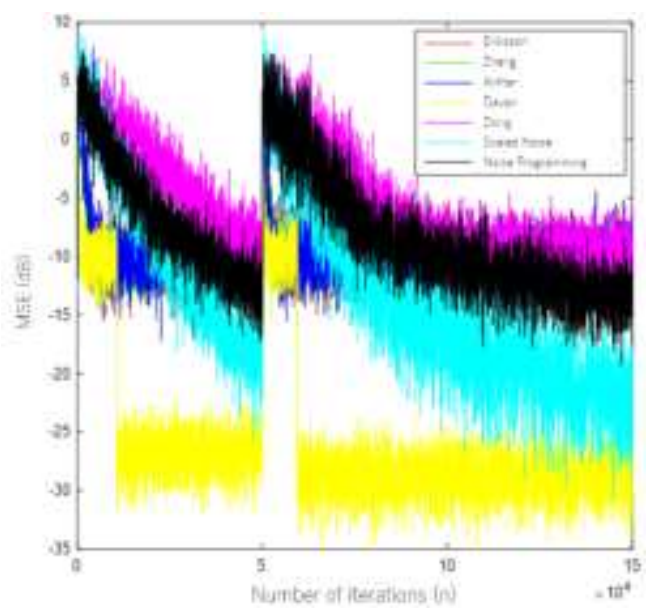

Fig. 11 Simulation results for Case 2, MSE. 


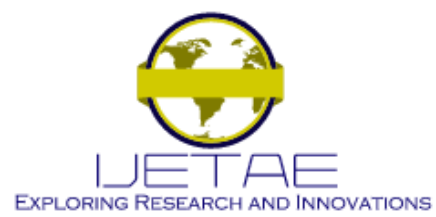

International Journal of Emerging Technology and Advanced Engineering

Website: www.ijetae.com (E-ISSN 2250-2459, SCOPUS Indexed Journal, Volume 10, Issue 12, December 2020)

In practice, the disadvantage of using the training signal is that adding it to the system reduces attenuation. Currently, to increase attenuation, methods are proposed that include training signals that not only increase attenuation, but also do it fast. Therefore, in a real application, not only are adaptive algorithms required in actual noise attenuation, but also the analysis of other noise sources must be considered. Nyquist noise, that is noise generated by the microphone and by the speaker, is an example of how other physical phenomena affect the expected performance of the system. Thus, it is required to incorporate the Nyquist noise analysis and determine its effect on the performance of the ANC system. Therefore, it is not enough to propose new algorithms, the physical implementation must be efficient, and the selected hardware must be properly used.

\section{Table III}

Simulation RESULTS

\begin{tabular}{|c|c|c|c|}
\hline & \multicolumn{2}{|c|}{ Case 1 } & \\
\hline Proposal & $\mathbf{\Delta S} \mathbf{( d B )}$ & $\mathbf{M S E} \mathbf{( d B )}$ & Iterations (n) \\
\hline Eriksson & -37.97 & -10.44 & $2.00 \times 10^{3}$ \\
\hline Zhang & -55.69 & -10.55 & $2.00 \times 10^{3}$ \\
\hline Akhtar & -43.12 & -10.56 & $1.75 \times 10^{3}$ \\
\hline Davari & -40.37 & -30.19 & $1.20 \times 10^{4}$ \\
\hline Scaled Noise & -27.92 & -29.16 & $1.70 \times 10^{4}$ \\
\hline $\begin{array}{c}\text { Noise } \\
\text { Programming }\end{array}$ & -36.72 & -29.86 & $6.00 \times 10^{4}$ \\
\hline Dong & -49.01 & -10.54 & $5.00 \times 10^{4}$ \\
\hline \multicolumn{4}{|c|}{ Case 2 } \\
\hline Proposal & $\Delta$ S (dB) & MSE (dB) & Iterations (n) \\
\hline Eriksson & -41.19 & -9.86 & $4.0 \times 10^{3}$ \\
\hline Zhang & -43.75 & -9.91 & $4.0 \times 10^{3}$ \\
\hline Akhtar & -41.23 & -9.91 & $4.0 \times 10^{3}$ \\
\hline Davari & -41.05 & -29.01 & $8.7 \times 10^{3}$ \\
\hline Scaled Noise & -35.33 & -25.10 & $1.0 \times 10^{5}$ \\
\hline $\begin{array}{c}\text { Noise } \\
\text { Programming }\end{array}$ & -29.66 & -14.77 & $1.0 \times 10^{5}$ \\
\hline Dong & -43.18 & -9.85 & $4.5 \times 10^{4}$ \\
\hline
\end{tabular}

In order to offer a higher speed proposal convergence, with an error in attenuation equivalent to the proposed Davari, and of less mathematical complexity is that the authors propose algorithms (here-in-after called Lara I and Lara II) for the online estimation of the second CC in a system ANC. Our proposals uses an ECLMS algorithm and includes scaling of the auxiliary noise source.

Lara I uses an ECLMS algorithm, which has a faster convergence speed and less computational complexity. It is based on the encoding of the error and reduces floatingpoint operations. Lara I also uses a scaling of the auxiliary noise source simply by delaying the error signal.
In order to improve the modeling value of the second $\mathrm{CC}$, it is performed a variation to Lara I's proposal, and it is programming the source auxiliary noise (this variant is Lara II). Then, in order to compare the proposed methodologies, a behavioral simulation was carried out, and the same strategies already mentioned were used [1]. The results obtained, for case 1, are shown in Fig. 12, where Zhang's proposal presents the better modeling of the second CC with an error of $-54.45 \mathrm{~dB}$. While Lara I and Lara II have a modeling error of $-11.09 \mathrm{~dB}$ and $-32.74 \mathrm{~dB}$, respectively. Another simulation is shown in Fig. 13, where our proposals reach the best MSE values with $-31.45 \mathrm{~dB}$ and $-31.65 \mathrm{~dB}$ for Lara I and Lara II, respectively. Based on these results, it can be said that the proposed methods have high speed of convergence [1]. The ECLMS algorithm is indeed simple, of less mathematical complexity, and suitable for its digital implementation in a DSP.

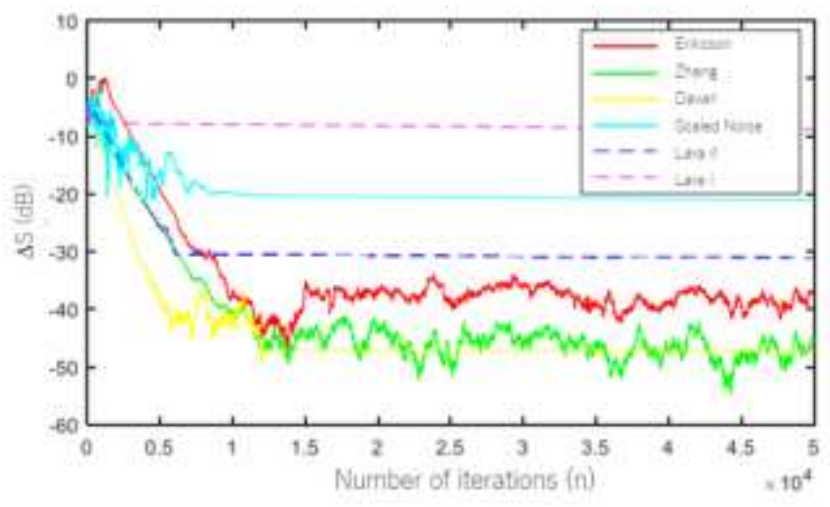

Fig. 12 Simulation results for Case 1, modeling error.

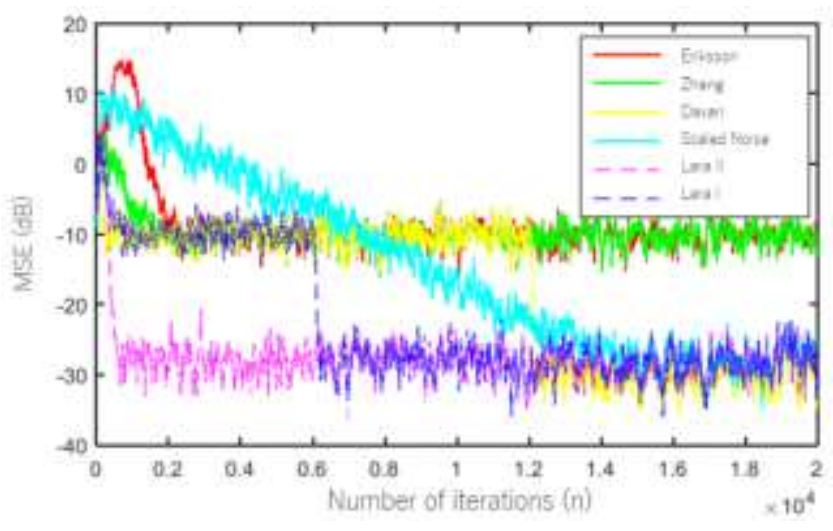

Fig. 13 Simulation results for Case 1, MSE. 


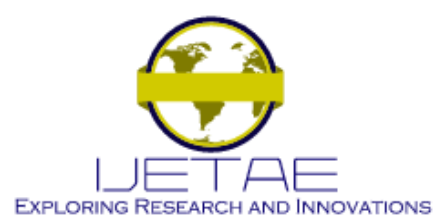

\section{International Journal of Emerging Technology and Advanced Engineering Website: www.ijetae.com (E-ISSN 2250-2459, SCOPUS Indexed Journal, Volume 10, Issue 12, December 2020)}

\section{CONCLUSIONS}

The analysis of the methods proposed in the literature to implement an active noise cancellation system was presented. A comparison was made to show which proposals are the most relevant and how they behave in the face of narrow band noise, broad band noise and in the face of sudden variations in CC. It is concluded that the adaptive algorithms affect the speed of convergence of the system and also the value of the error obtained (it decreases as the attenuation factor increases). It should be noted that in ANC systems the online estimation of the secondary CC is very important, since it allows the system to adapt its coefficients to environmental changes and due to the aging of the acoustic transducers, among other phenomena [20]. Therefore, the better the estimate of the secondary $\mathrm{CC}$, the greater the attenuation will be obtained in the system.

What is new? Recently some proposals have been published related to how to mitigate the noise added by random noise. Those proposals correspond to scaled noise [21], auxiliary noise power programming and more of the Davari method. All of them have a common disadvantage, and it is a low speed of convergence and an increase in the complexity of the architectures. However, a proposal that deserves attention is that of M. T. Akhtar [22]. He proposes using gain controlled probe noise mixed with the cancellation signal. In practice, that gain-controlled noise is adjusted to the desired value so that high-amplitude noise is generated. Furthermore, this feature improves the convergence time in the cancellation path estimation. As the ANC system converges, the level of the noise signal is reduced and a good performance of the purpose of reducing noise is achieved, but in a strict sense that proposal is similar to the scaled noise method [17], [21] and also to the method proposed by Davari [d]. Other proposals use a step size controller to improve both convergence stability and noise reduction in the ANC [22]-[24] system. The step size scaler prevents incorrect updating of weights by making the step size small when impulsive noise is detected in the system. This adds complexity to the architecture. Finally, one more proposal suggests modeling the secondary path without auxiliary noise as long as the noise to be controlled is not a line spectral property [25]. In other words, if that property is not fulfilled, the proposal is not useful.

Based on the results of the analysis, in this paper a new algorithm based on both Lara-I and Lara-II is proposed, which is suitable for hardware implementation. Our choice is the TAdsp28335 card. With it, two purposes are defined: to develop a low-cost system and to implement the optimal processing algorithms for real-time operation.

\section{Acknowledgments}

The authors thank CONACyT (Mexico) for the financial support granted through project 169660. EOLA thanks CONACyT for the PhD scholarship received.

\section{REFERENCES}

[1] Lara-Alemán E. O., 2020 Diseño e implementación de un sistema ANC para cancelar ruido de banda angosta en un rango de frecuencia de $100 \mathrm{~Hz}$ a $1.5 \mathrm{kHz}, \mathrm{PhD}$ Thesis, Cinvestav-Guadalajara

[2] Widrow B., 1966 Adaptive filters I: Fundamentals, Stanford Electronics Laboratories Technical Report 6764-6.

[3] Troshin A. G., Cha S. and Kim D., 2011. Evaluation and parametric study of LMS algorithms family for active noise control barriers, In International conference on Control, Automation and Systems.

[4] Eweda E., Comparison of RLS, LMS, and sign algorithms for tracking randomly time-varying channels, in IEEE Transactions on Signal Processing, 42 (Nov. 1994) 2937-2944.

[5] Eweda E., Transient performance degradation of the LMS, RLS, sign, signed regressor, and sign-sign algorithms with data correlation, in IEEE Transactions on Circuits and Systems II: Analog and Digital Signal Processing, 46 (Aug. 1999) 1055-1062.

[6] Merched R., Extended RLS lattice adaptive filters, in IEEE Transactions on Signal Processing, 51 (Sept. 2003) 2294-2309.

[7] Xiao Y., Ma L., Khorasani K., A. Ikuta and Li Xu, 2005. A filtered$X$ RLS based narrowband active noise control system in the presence of frequency mismatch, In IEEE International Symposium on Circuits and Systems.

[8] Iman Tabatabaei A., Waleed H. A., 2011. "FxLMS-based Active Noise Control: A Quick Review”, In Proc. of Asia-Pacific Signal and Information Processing Association.

[9] Rupp M. and Sayed A. H., 1995. Two variants of the FxLMS algorithm, In Proc. of Workshop on Applications of Signal Processing to Audio and Accoustics.

[10] Lopez-Gaudana E. et al, 2008. A Hybrid Active Noise Cancelling with Secondary Path Modeling, In Proc. of 51st IEEE Midwest Symposium on Circuits and Systems.

[11] Burgess C., Active adaptive sound control in a duct: A computer simulation, J. Acoustical Soc. Amer., 70 (Sept. 1981) 715-726.

[12] Eriksson L. J. and Allie M. C., Use of random noise for on-line transducer modeling in an adaptive active attenuation system, J. Acoust. Soc. Amer., 85 (Feb. 1989) 797-802.

[13] Kuo S. M. and Morgan D. R., 1996 Active Noise Control Systems Algorithms and DSP Implementations, John Wiley \& Sons.

[14] Zhang M., Lan H., Ser W., Cross-updated active noise control system with online secondary path modeling, IEEE Trans. Speech Audio Process., 9 (2001) 598-602.

[15] Akhtar M. T., Abe M. and Kawamata M, A new variable step size LMS algorithm-based method for improved online secondary path modeling in active noise control systems, in IEEE Transactions on Audio, Speech, and Language Processing, 14 (March 2006) 720-726.

[16] Davari P., Hassanpour H., Designing a new robust on-line secondary path modeling technique for feed forward active noise control systems, in Signal Processing, 89 (June 2009) 1195-1204.

[17] Liu J., Xiao Y., Sun J. and Xu L., Analysis of Online SecondaryPath Modeling With Auxiliary Noise Scaled by Residual Noise Signal, in IEEE Transactions on Audio, Speech, and Language Processing, 18 (Nov. 2010) 1978-1993. 


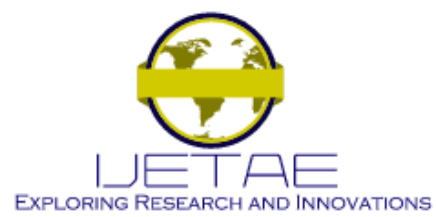

International Journal of Emerging Technology and Advanced Engineering Website: www.ijetae.com (E-ISSN 2250-2459, SCOPUS Indexed Journal, Volume 10, Issue 12, December 2020)

[18] Sun Ch. et al, A New Online Secondary Path Modeling Method with An Auxiliary Noise Power Scheduling Strategy for Narrowband Active Noise Control Systems, Applied sciences, 7 (Nov. 2017) 114.

[19] Kim D. W. and Park P., 2019. A novel active noise control system with online secondary-path filter based on a step size controller, In 12th Asian Control Conference.

[20] Lara-Alemán E.O. and Sandoval-Ibarra F., Accepted paper at 4th International E-Conference on Advance in Engineering, Technology and Management, January $21^{\text {st }}, 2021$

[21] Bai T., Wang Z., Xiao Y., Ma Y., Ma L. and Khorasani K., 2019. A multi-channel narrowband active noise control system with simultaneous online secondary- and feedback-path modeling, in IEEE Asia Pacific Conference on Circuits and Systems.

[22] Akhtar M. T., 2019. A Fully Adaptive Feedback ANC System Employing Online Estimation of the Cancellation Path, in IEEE 62nd International Midwest Symposium on Circuits and Systems.

[23] Manzano E. A. and Lescano E. J. L., 2019. Optimal Step Size of Delayed FxLMS Algorithm for Multi-frequency Active Noise Control Systems, in International Conference on Information Systems and Computer Science.

[24] Kim D. W., Lee M. and Park P., 2019. A robust active noise control system with stepsize scaler in impulsive noise environments, in Chinese Control Conference.

[25] Woo Kim D. and Park P., 2017. Online secondary path estimation in active noise control systems using a scheduled step size algorithm, in $11^{\text {th }}$ Asian Control Conference.

[26] Hu M. and Lu J., 2020. Active Control of Line Spectral Noise with Simultaneous Secondary Path Modeling Without Auxiliary Noise, in IEEE International Conference on Acoustics, Speech and Signal Processing.

\section{AUTHOR'S PROFILE}

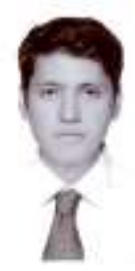

E. O. Lara-Alemán received the BS degree in Mecatronics from Instituto Tecnológico de Celaya (ITC), MSc and DSc degrees in Electronics from Centro de Investigación y de Estudios Avanzados (CINVESTAV), Guadalajara Unit, Mexico, in 2013, 2015 and 2020, respectively.

His research interests are on the efficient design and implementation of digital signal processing algorithms, digital systems design and analysis of algorithms for canceling acoustic noise.

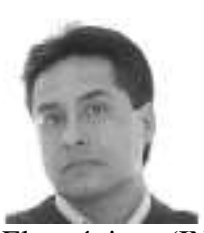

F. Sandoval-Ibarra received the BS degree in Physics Electronics from Facultad de Ciencias, Universidad Autónoma de San Luis Potosí (UASLP), San Luis Potosí, Mexico, in 1988, and MSc and DSc degrees in Electronics from Instituto Nacional de Astrofísica, Óptica y Electrónica (INAOE), Puebla, Mexico, in 1991 and 1998, respectively.

He was a researcher at Laboratorio de Microelectrónica, INAOE, from 1991 to 1997 , where he developed wet-etching techniques for designing silicon-based microsensors. In 1997, he was with CNM-Bellaterra (Spain), as a visiting researcher being involved in the development of surface micromachining techniques to design a fully-integrated microphone. He was a fulltime Professor at Instituto Tecnológico de Puebla (ITP), in 1998. Since 1999, he has been a Professor and Researcher at Cinvestav, Guadalajara Unit (GU). From 2015 to 2020, he was the Coordinator of the Electronic Design Group at GU. His research areas include the design of low-noise analog integrated CMOS circuits, mixed-mode circuits design for RFID applications and the physical modelling of electronic noise using digital signal processing algorithms. He is currently working on the design of A/D converters based on Hybrid $\Sigma \Delta$ Low-Pass Modulators for multi-standard applications.

Dr. Sandoval-Ibarra is a member of Sistema Nacional de Investigadores (SNI), Mexico.

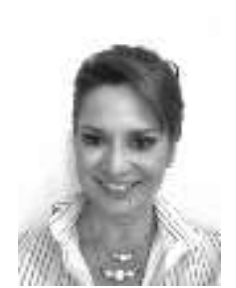

Susana Ortega-Cisneros received the Engineering degree in Electronics and Communications Engineering from the University of Guadalajara, Guadalajara, Jalisco, Mexico, in 1990, the M.Sci. degree in solid state electronics from the Center for Research and Advanced Studies (Cinvestav), National Polytechnic Institute, Mexico City, Mexico, in 1995, and the Ph.D. degree in computer science and telecommunications from the Autonomous University of Madrid, Spain, in 2005.

Since 2010, she has been with the Center for Research and Advanced Studies (Cinvestav), National Polytechnic Institute, Guadalajara, Jalisco, Mexico. She is involved in the design of digital architectures based on FPGAs, digital signal processors (DSPs), and microprocessors. Her current research interests include digital control, self-timed synchronization, electronic systems applied to biomedicine, embedded microprocessor design, digital electronics, custom DSPs in FPGAs, and, recently, remote sensing applications.

Dra. Ortega-Cisneros is a member of Sistema Nacional de Investigadores (SNI), Mexico. 